Marquette University

e-Publications@Marquette

Marketing Faculty Research and Publications

Business Administration, College of

$1-1-2008$

Understanding Behavioral Sources of Process

Variation Following Enterprise System

Deployment

Elliot Bendoly

Emory University

Mark Cotteleer

Marquette University, mark.cotteleer@marquette.edu

Post-print.

Journal of Operations Management, Volume 26, No. 1 (January 2008), DOI: 10.1016/

j.jom.2007.03.002. 


\title{
Understanding Behavioral Sources of Process Variation Following Enterprise System Deployment
}

\author{
Elliot Bendoly ${ }^{\mathrm{a}, *}$, Mark J, Cotteleer ${ }^{\mathrm{b}, 1}$
}

\begin{abstract}
This paper extends the current understanding of the time-sensitivity of intent and usage following large-scale IT implementation. Our study focuses on perceived system misfit with organizational processes in tandem with the availability of system circumvention opportunities. Case study comparisons and controlled experiments are used to support the theoretical unpacking of organizational and technical contingencies and their relationship to shifts in user intentions and variation in work-processing tactics over time. Findings suggest that managers and users may retain strong intentions to circumvent systems in the presence of perceived task-technology misfit. The perceived ease with which this circumvention is attainable factors significantly into the timeframe within which it is attempted, and subsequently impacts the onset of deviation from prescribed practice and anticipated dynamics.
\end{abstract}

\section{Introduction}

What gives rise to variation in operational processes? This relatively broad question can be addressed from a number of perspectives. First and fundamentally, organizations consist of a wide range of distinct processes, including those that transfer materials and information and those that implement and/or document product and organizational design changes. Such procedural scope provides an implicit source of variation in activities and outcomes (Garvin, 1998). Second, variation may exist across a set of relatively similar processes or, at the extreme, even within a single generalized process (e.g., billing) (Frei et al., 1999). Since any process may be the responsibility of a number of individuals (e.g., as parallel processors of concurrent tasks), we would expect to see at least some variation in the amount of time and effort spent.

Numerous issues might influence the extent of process variation. Some may be due to differences in skills or specific task instances. Such variation may be beyond the control of individual workers. Other issues may not be. For example, the extent to which an individual follows a suggested or prescribed set of steps or rule-structure (Saxberg and Slocum, 1968). Some workers may be averse to deviating from prescriptions, while their co-workers may instead seek out such departure (Boyce, 2001). If a rule-structure is relatively non-binding, we expect 
that as average discomfort with the structure increases the range of practices used by workers to execute that process will also increase. We depict this by contrasting the range of practices used in two processes, each differing only by the level of average discomfort with prescribed rule-structures (see Fig. 1).

However, the above conceptual unpacking of variation in individual practice remains somewhat naïve on at least one major front. For many processes, individuals may not have freedom to choose their approach to work. Workers are often constrained by the range of permissible or feasible work practices, as mandated by strong rule-structures imposed on them. These strong rule-structures may be organizational (e.g., linking compliance to performance evaluation) or technical in nature (e.g., other processing options are not supported by the IT). With specific reference to technically imposed strong rule-structures, many firms have adopted IT with the intent to standardize work practices and promote greater consistency. Enterprise resource planning (ERP) systems provide an excellent example of a type of IT deployed with this intent (Davenport, 1998; Cotteleer, 2006). The implication of such strong role-structures in suppressing process variation, at least in the short-term, is illustrated in Fig. 2.

Our study focuses on how organizations and employees react to rule-structures that accompany the implementation of large-scaled ERP, an IT category known for rule-structure rigidity. We contribute to the literature on IT-supported benefits by considering both short- and long-term intention, and variation in work associated with alternate levels of operational process and IT-protocol misfit. We simultaneously examine the role of alternate levels of ease by which IT can be circumvented. Although theory and anecdotal evidence suggest complex contingencies may arise between these kinds of operational and information technology issues, there is a paucity of controlled experimentation that explicitly attempts to account for the interaction of misfit, ease of circumvention and time on intended behavior. By theorizing with regard to, and empirically comparing, short- and long-term reactions we contribute to the understanding of sources and timing of trends in process variation following IT implementation.

We begin by reviewing the related literature in support of a theoretical framework for shifts in process variation. This is followed by a discussion of two field cases in which trends in variation of operational processing markedly differ. Hypotheses based on our theoretical framework and this case evidence provide the backdrop for an experimental design in which we study the reactions of managerial-users to one of four alternate contexts that differ in their levels of task-technology misfit and ease of system circumvention. The results of our controlled experiment show a strong intention on the part of managers to circumvent implemented systems when faced with strong task-technology misfit. Perceived ease of circumvention factors 
significantly into the time frame of the intended circumvention. Our findings emphasize the need for implementers to attend not only to ensuring real fit between technology and operational processes, but also to the management of perceptions relating to long-run task-technology misfit.

\section{Theoretical background}

ERP systems are multifaceted and wide-reaching in their scope of process and organizational influence (Mabert et al., 2003; Stratman and Roth, 2002; Davenport, 2000). They are often touted as a means for (1) standardizing and centralizing specific processes, hence reducing variation in processing practice, time, and error; (2) increasing the transparency of process work and outcomes across an organization and; (3) increasing the predictability of processes for operational and strategic planning purposes as a result of these first two capabilities (Bendoly and Jacobs, 2005; Jacobs and Bendoly, 2003; Rabinovich et al., 2003; McAfee, 2002). Regardless of the form and function of the ERP, it is clear that the imposition of the system's codified protocols on work processes is likely to have some impact on the form and function of the organization, and on the behavior of those in the organization charged with its use (Cotteleer and Bendoly, in press). As with other types of IT, an understanding of ERP benefits cannot be sufficiently gained without an appreciation of these organizational impacts (Barki and Pinsonneault, 2005; El Sawy and Majchrzak, 2004; Brynjolfsson and Hitt, 2003; Brynjolfsson et al., 2002; Griffith et al., 2002).

Beyond changing the way people work, ERP may also impact how people feel about the work they do. Furthermore, upper management's perspective on an appropriate fit between processing "needs" and "prescribed" protocols may be different from that of the individual users (Soh et al., 2003; Griffith et al., 1999). This is a critical issue since even within the context of ERP (where procedural protocols are fairly well-structured) alternative approaches to processing may exist. Individual tendencies to comply with prescribed usage (e.g., that in which they have been trained during system implementation), and therefore the ways in which said usage will influence organizational form and function, may be difficult to predict when non-prescribed alternatives are clearly available (Boudreau and Robey, 2005). Certainly, given the shared perspective of much

of the ES literature - that it has a strong standardizing influence on processes and outcomes (c.f., Davenport, 1998; Lee and Lee, 2000; Scheer and Habermann, 2000; Bendoly, 2001; Light et al., 2001; Razi and Tarn, 2003; Bendoly and Kaefer, 2004; Seely Brown and Hagel, 2004; Trott and Hoecht, 2004) - investigations into the factors influencing such deviations are warranted.

Several bodies of literature suggest that individuals will seek out alternatives when they are uncomfortable with prescribed ERP protocols. Most fundamental is the organizational 
change literature (Oreg, 2003; Dougherty, 2001; Gersick and Hackman, 1990; Rogers, 1983) which suggests that change in general (i.e., regardless of users' perceptions of fit) prompts a search for procedural alternatives akin to those in place prior to implementation. While this view focuses on the congruence between the "extent of" and "resistance to" change in general, it nevertheless represents a potentially valuable baseline for considering variation in use (i.e., some users may inherently be more averse to imposed procedural changes than others).

In contrast, several alternative viewpoints take more contingent approaches to explaining variation in use (Umanath, 2003; Weill and Olson, 1989). These include theories such as reasoned action (TRA) (Fishbein and Ajzen, 1975) which, as applied in the technology acceptance model (TAM), has been focused on how work behavior may be contingent on both the perceptions of ease of use and usefulness of an IT. TAM has clearly enjoyed popularity over the years and continues to provide an effective mechanism for capturing certain forms of variation in use (c.f. Venkatesh, 1999; Mahmood et al., 2001). More recently, TAM has been applied in specific attempts to model ERP use intentions (Amoako-Gyampah and Salam, 2004). Although this work presents a generalization of the specific ERP form and function, its findings nevertheless suggest that intentions, even with respect to highly structured protocols, may be sensitive to user beliefs.

Some have argued that TAM's focus has a failing in that it insufficiently captures the work context of the IT (Legris et al., 2003; Goodhue and Thompson, 1995). Although contextual extensions to TAM have been proposed, many researchers have instead opted to apply task-specific models of "use" in their research, as proposed by the task-technology fit (TTF) literature (Dishaw and Strong, 1999; Goodhue, 1998; Goodhue, 1995), or more user context control-specific applications of the theory of planned behavior (TPB) (Ajzen, 1991). Studies based on these models suggest that contextually focused issues such as "control" (Zammuto and O'Connor, 1992) and "fit with existing process" can have significant impact on the form of intended use and dependent measures of performance (c.f. Bendoly and Jacobs, 2004; Van Stijn and Wensley, 2001). Although detractors have criticized the paucity of evidence in support of the virtues of such models as improvements on TRA and TAM (e.g., Venkatesh, 1999; Venkatesh and Davis, 2000), there is a clear competing camp that has attempted to emphasize the empirical support of their contributions (Beaudry and Pinsonneault, 2005; Chau and Hu, 2001; Dishaw and Strong, 1999; Taylor and Todd, 1995; Taylor and Tucker, 1989; Mathieson, 1991). In fact, more recent incarnations of TAM (e.g., the unified theory of acceptance and use of technology or UTAUT) have taken calculated steps to incorporate more context specific issues (Venkatesh et al., 2002, 2003). 
We share this latter sentiment and see value in an extended contingency approach to deconstructing variation in use that accounts for not just issues of technology fit and user control, but also contingency on the time-frame in which use is characterized. As such, our view is representative of traditional valence-instrumentality-expectancy (VIE) frameworks (Vroom, 1964; Weiss et al., 1999). In our particular focus on the "range of" departures from prescription, we view valence as relating to the attractiveness of system circumventions and alternatives to prescribed use (Schultze and Orlikowski, 2004; Weick, 1998; DeSanctis and Poole, 1994).

Traditional facets of expectancy and instrumentality relate to both the contingencies represented by time, and to ease of action (in our case, the ease with which circumvention can be achieved). One can argue that contexts in which ease is high are likely to raise expectations that circumvention-based resolution of "misfit" may be readily enjoyed. In relation to cognitive perspectives of resolutions in perceived misfit, such an active "resolution" mechanism would be akin to rotating the "actual activity" (performance) axis toward the line of expectation (George, 2003; Szajna and Scamell, 1993; Robbins, 1986; Festinger, 1957). One might anticipate such "rotation" to continue until perceived misfit is no longer a strong enough force to drive the pursuit of additional work-arounds. Such stopping conditions may exist either because practice has sufficiently approached work-expectations and/or because expectations themselves have been altered (Van Stijn and Wensley, 2001).

When ease of circumvention is low, and while perceptions of misfit continue to provide a high valence for non-prescribed use, other factors may be instrumental in achieving such resolution-"time," and implied experience with the system, being an obvious candidate (Robey et al., 2002; Tyre and von Hippel, 1997; Stein and Vandenbosch, 1996; Tyre and Orlikowski, 1994). The incorporation of "time" as a critical element of the VIE perspective is not new (e.g., Mayes, 1978). More broadly, the time-factor represents a relatively standard element of accepted frameworks relating to innovation phenomena within firms (Narayanan, 2001; Rogers, 1983). Notwithstanding, discussions of the instrumental role of time in the emergence of deviations from "ideal" use are more rare (Orlikowski, 2000 provides an exception). Simply stated, as people gain experience with system use, they also gain experience with how to misuse it, for better or worse.

Such phenomena is not sufficiently captured in the majority of IT literature, and is particularly underexplored in literature focusing on phases of implementation (e.g., adoption, adaptation, acceptance, use) where the standard codification approach is that of a lock-step mechanism with discrete points of reference. More realistic is a view which recognizes that adaptation and acceptance phenomena take place in parallel, with significant feedback interweaving the two mechanisms. Given Beaudry and Pinsonneault's (2005) recent arguments 
regarding coping in new innovation implementations, it is conceivable that initially observable levels of user acceptance may be very different from those observed at later points-as adaptation continues. Theoretically, this represents a split from the more traditional phase-reference models investigated to date.

It is also possible that the long reliance on phase-reference frameworks may be a partial cause for some of the confusion in the use of the term "ironic appropriation" as directly synonymous with instability (c.f. Gopal et. al's discussion 1992-1993, p. 47). Few would argue that isolated observations of faithful appropriation can give way to observations of ironic appropriation depending on issues such as user attitudes. However, it is conceivable that stable appropriation could also be established given an attitude-driven consensus on misuse (i.e., "ironic" appropriation). Approaching such stability at an "ironic" equilibrium would require considerable search and hence variation in use, across users and over time. In fact ultimately, and regardless of the nature of the appropriation, the process of equilibrium development remains contingent on time, perceived suitability of "faithful appropriation" (i.e., fit) and the ability to overcome barriers to alternate use-an ability that itself may change over time.

Fig. 3 characterizes the contingent aspects of each of the three issues of perceived contextual misfit, ease of circumvention (EOC) and time-frame as they relate to potential departures from prescribed actions (i.e., ERP-imposed protocols in our case). The depiction of practice here, represented by the position of the small rectangle in each quadrant, relates to our earlier view of variability in practice (e.g., Figs. 1 and 2 presented earlier). In the diagram, the further practice strays from the prescribed (i.e., the further right it is positioned) the greater the range of practice to be expected-a range that incorporates both those averse to, or incapable of, circumvention as well as those most willing and able to avail themselves of circumvention opportunities.

We believe our contribution to the post-implementation use and ERP deployment literatures stems from our insight into contingencies relevant to contexts in which new technology-driven protocols: (1) represent a perceived misfit with operational procedures and; (2) are imposed as strong rule-structures whose limitations on alternatives in practice may vary over time. Through our chosen theoretical lens and empirical evidence, we hope to provide a means of clarifying the IT and organizational form/functional issues that contribute to trends in practice variation over time in organizations adopting new technologies.

\section{Cases and hypotheses}

We draw on a pair of case studies as a first step to illustrating contingent time-delayed 
effects that may follow implementation. The cases contrast examples of sustained versus non-sustained compliance with IT-supported protocols. Both cases represent implementations of the same ERP package in high-tech companies. The cases were selected based on their IT commonalities so as to minimize endogenous issues, and to emphasize distinctions in operating dynamics.

Development of the case studies followed the guidance of Yin (1994), our goal being "to develop pertinent hypotheses and propositions for future inquiry" (p. 5). The cases rely on three complementary sources of information to assess the context and results of the firms' ERP initiatives. The first two facilitate an understanding of the implementation context. These consist of (1) an archival review of implementation and change documentation, as well as (2) more than $40 \mathrm{~h}$ of interviews with firm managers, project participants, and system users. The third source of information consisted of the firms' operational data collected over the duration of the ERP initiative. This data served as the foundation for the quantitative summary presented here.

\subsection{Case 1: TECH-peripherals division}

Peripherals division (PD) was a $\$ 600$ million division of TECH Manufacturing, a $\$ 2$ billion, US-based manufacturer of computer and electronic equipment. PD operated in 12 geographic regions, encompassing more than 20 countries. In the late 1990s, PD participated with other divisions in the rollout of ERP applications with the goal of enabling growth and simplifying a morass of legacy IT applications. Anticipated benefits included faster order fulfillment, simplified financial processes, and global logistical coordination.

Despite a goal to minimize customization, managers recognized that local users might argue they faced unique circumstances requiring differences in operational activities. PD addressed these situations by employing teams of business experts to analyze local requirements and determine whether a misfit truly existed, or whether it was merely perceived based on traditional business practices. In some cases, adaptation seemed justified. For example, the firm developed a "bolt-on" application to translate customer documentation into local languages. However, in a majority of cases conflict did not seem to arise from true business need. Here, extensive discussion with local representatives ensued in order to communicate the need for standard system supported protocols.

Following implementation, employees reported that the opportunity to gain experience with a new information system complemented the operating processes that had been in place. Employees claimed that a repetition of process steps enhanced comfort with the execution of functional tasks. A process of continuous learning lasted as long as 3 months. "We definitely got 
better as time went on," reported one local user. "When you first started, it seemed like it took forever to get an order done. Our trainers would say, 'The more you use it, the better you get.' So you got in there did it, and you just got quick at it."

"Super-Users" provided additional local post-implementation support. These system experts - drawn from the ERP deployment team - provided assistance to those encountering difficulties that could be viewed as conflicts between processing needs and system capabilities. Super-users provided a kind of authoritarian hierarchy that helped regulate the use of system-protocols and discourage circumvention attempts. Management believed that improvements in both efficiency and predictability enabled by the ERP deployment would become a sustainable characteristic of its business. User feedback supported this belief.

Supporting evidence also appeared through a comparison of associated performance shifts in order lead-time across PD's operating divisions. Fig. 4 summarizes these observations, with average lead-times scaled to the pre-system benchmark for comparability with the next case. In the first month following deployment, average lead-times showed a significant decrease (from the pre-system average of 10.1 days to an average of 7.2 days). Lead-time variations also saw a significant decrease (from a standard deviation of 2.1 days to 1.2 days). Given that the system's standardized protocols could assist in and stabilize the order-processing task, both of these results were anticipated by TECH managers. In particular, managers believed that decreased variability indicated a general adoption of the standardized system protocols. Most important, given TECH's interest in greater supply chain predictability, the sustained decrease in process variation that appeared over the following 24 months was seen as particularly valuable for future planning purposes and for fostering the perception of TECH as a reliable supply chain partner.

\subsection{Case 2: Tristen incorporated}

Tristen, Inc., a $\$ 4$ billion manufacturer of computer components, operated in three autonomous regions, maintaining more than 20 globally distributed sales offices. Like PD, Tristen sought to minimize system modification and to adhere to the process standards encouraged by the software. Also like PD, Tristen sought to provide more predictable service levels to global customers through centralization and process standardization across operating regions.

Tristen's deployment effort took place at the firm's North American headquarters. More than $95 \%$ of full-time project personnel originated in the North American region. This development approach resulted in the perception that managers from other regions had limited project input. One manager characterized the effect of the centralized deployment strategy by 
suggesting "if you did not send someone to the project, you were not represented, you were [expletive deleted)."

Despite challenges, Tristen significantly reduced variation relative to order lead time. Average lead-times fell by nearly 4 days following deployment-from approximately 11 days pre-deployment, to 7 days after the first month. Concurrent with these improvements (as in the TECH case) was a convergence in processing practice across the firm's operations. Lead-time variation showed a sizeable drop, again attributed to the imposition of standardized protocols, from a pre-system level of around 2 days to around half a day after the first month. However, in contrast to $\mathrm{TECH}$, despite continued lead-time improvements, initial reductions in variation were not sustained (See Fig. 5). Lead-time variation began to increase as early as the eighth month and gains disappeared altogether by the 24th month following deployment.

A number of issues might account for increases in regional variation. Divergence might simply have resulted from faster learning in some regions. However, if it is reasonable to assume a common level of regional performance is asymptotically approachable, then variation should be expected to fall as that point is reached. Such a fall was not observed during the 24-month study period. Alternately, regional differences in process requirements may have been so great that the expectation of process standardization was unrealistic. However, discussions with Tristen managers did not suggest that this was the case.

The common explanation for divergence in practice at Tristen had more to do with sustained behavioral reactions to the system change. As system users continued to feel discomfort with new protocols, their growing familiarity with the system promoted an understanding of how to circumvent those protocols. While some discovered and engaged in such tactics, others did not-due to either different levels of familiarity or perceived benefit of doing so. Differences in use could lead to idiosyncrasies in performance-the same idiosyncrasies the firm had original intended to reduce through ERP deployment.

That differences in valence, instrumentality and expectancy of circumvention tactics existed within Tristen does not seem to be simple speculation. Reports of system users supported their existence. Managers indicated that substantial site-level adaptation emerged soon after ERP deployment. This adaptation resulted from wide-spread, sustained perceptions of misfit between the systems and local process requirements, resulting in sustained circumventive behavior. In some cases, local adaptation involved the discovery of new functionality or alternate ways of accessing information. In others, local users changed system parameters to better-fit perceptions of how the business should function. Implementation team personnel were explicit about the fact that the opportunity existed to execute these changes. "We 
didn't really have a good process in place to make sure that there was consistency where there needed to be," reported one team member. "We had some issues specifically with the logistics sites. Once they got into the system they started making changes there wasn't a good process to prevent that from happening."

\section{i,}

\subsection{Research hypotheses}

The contrasting trends in practice observed in these two cases provide an interesting foundation for further investigation. In the TECH case, a stronger adherence to the standard centralized model, the deployment of knowledge and discipline-providing resources, and the deployment of resources to mitigate perceived local mismatches, led to higher levels of true acceptance and continued learning in line with operationally planned practice. In contrast, the Tristen case illustrates a situation in which perceived misfits between local requirements and a centralized ERP deployment led users to seek ways to circumvent the intended business model. Although a range of mechanisms might be proposed to account for these differences, in these particular cases the self-reports of managers focused on unanticipated behavioral phenomena at Tristen. As outlined earlier, such behavioral phenomena seem substantiated from a theoretical perspective.

Based on the suggestiveness of this case information, as well as our application of established contingency perspectives in the literature, we would expect that the greater the misfit between information system protocols and operational task requirements, the more likely that individuals will seek out actions to circumvent the information systems (Soh et al., 2000, Van Stijn and Wensley, 2001). In our research we further extend this claim by specifically proposing: Hypothesis 1a.

For a given level of perceived misfit, short-term intentions to circumvent will be greater when circumvention options are more apparent then when circumvention options are less apparent.

That is, we anticipate greater intention to immediately pursue workarounds when these workarounds are more apparent. This hypothesis directly represents the under-explored notion of ease-of-circumvention central to our contingency-based framework, as well as alludes to the potential roles of both misfit and time that will be considered next. A corollary hypothesis based on our understanding of process variation and its relationship to average and extreme levels of intended use, as discussed in reference to Fig. 1, is as follows:

\section{Hypothesis $\mathbf{1 b}$.}

For a given level of perceived misfit, the variation in short-term intentions to circumvent 
will be greater when circumvention options are more apparent then when they are less apparent.

The rationale for posing these initial hypotheses is to provide a partially isolated proving ground for the concept of ease-of-circumvention prior to testing the more complex contingency relations in which we are interested. In addition to the ability to observe phenomena suggested by Hypotheses $1 \mathrm{a}$ and $1 \mathrm{~b}$, we further hypothesize that time-delayed effort to deviate from system protocols will be particularly observable when the tactics are not immediately available in high misfit contexts. Subsequent hypotheses correspond to differences between the left and right lower cells in Fig. 3, with reference to the starting condition represented in Fig. 2. That is:

\section{Hypothesis 2 a.}

At higher levels of perceived misfit, differences between long-term and short-term intentions to circumvent will be greater when circumvention options are less apparent.

The process-variation corollary is as follows:

\section{Hypothesis 2b.}

At higher levels of perceived misfit, differences between the variation in long-term and short-term intentions to circumvent will be greater when circumvention options are less apparent.

In short, we propose that in contexts where perceived misfit is high and circumvention appears to be easy, users will immediately seek to circumvent the implemented IT. If these circumventions are maintained, then the drive for additional circumventions may be reduced. On the other hand, users will persevere in their attempts to subvert where circumvention does not appear to be immediately attainable, provided that their perceptions of misfit supply the needed impetus. In marshalling a growing knowledge of the system over time, long-run circumvention may be achieved and hence intentions may become a reality, as in the Tristen case. By corollary, these greater levels of circumvention are likely to give rise to greater levels of variation in practice, again as realized in the Tristen case. Such a conclusion with respect to intent and its implications on practice would be important, since it would ultimately suggest that organizations seeking conformance to systems "as implemented" should continue to promote an understanding of (or at least perceptions of) the system's fit to local conditions. As in the TECH case, it may be insufficient to rely solely on system protocols to enforce conformance in post-implementation contexts.

\section{Experimental design}

The wide-array of operational processing differences among large corporations makes firm-level units of analysis impractical when attempting to assess the role IT misfit, ease-of-circumvention and time on patterns of intended use (Brynjolfsson and Hitt, 1996). 
Therefore, a micro-level investigation into human behavior can be more effective in describing the underlying operational phenomena that give rise to the differences in use ultimately observed at these higher-levels. The ability to observe temporally distinct intentions in a controlled experimental setting would add credence to a contingency-based model of the post-implementation reemergence of process variation. If clearly observable at the level of the individual, the implied potential for extension to higher-levels of analysis could prove a substantial contribution in itself.

With such an interest in mind, we now outline a controlled experimental investigation focused on describing the contingency-driven distinctions that can arise between intended short-term and long-term behavioral reactions to task-technology misfit. In analogy to the Tristen case, our context of choice is that of an ERP implementation. Existing theory and anecdotal evidence lead us to believe that distinctions in intended use over time will be significantly and contingently linked to the extent of misfit and the ease by which system-supported protocols can be circumvented.

Our experiment involves a controlled manipulation of context with the dual goals of reducing the risk of common-source, common-method bias, and of retaining adequate sample size for evaluation. Our research design contrasts those that rely on self-reports of on-the-job contexts where uncontrolled issues can complicate the interpretation of results. Specifically, we condition subjects by providing them with one of four case scenarios and focus their reactions to each. Such conditioned experimental designs are common in behavioral studies of operating environments and have proven valuable in providing insights into dynamics overlooked in less rigorously controlled studies (c.f. Bendoly and Swink, 2007; Schultz et al., 1999; Bachrach et al., 2001).

The orientations of the four contextual scenarios (i.e., treatments) used in our experiment are outlined in Table 1. The anticipated time-specific reactions hypothesized in the last section are also outlined in each cell of this table. Table 1 puts into words the nature of the theorized dependency of "intended use," and variation thereof, on the contingencies of misfit, ease-of-circumvention and time earlier conceptualized in Fig. 3.

Four modular case descriptions were developed in order to examine whether the time-specific reactions predicted by our framework could be observed in actual intended behavior. Each case began with a description of either a high- or low-misfit scenario. Each ended with a description of either a high or low ease-of-circumvention condition (see Appendix A). The verbiage used in these stimuli was based on excerpts from our case transcripts. To ensure unambiguous comparisons in later analysis, each case described the implementation of a 
standardized enterprise-wide order processing system. Thus sources of misfit came only from the differences in operational order processing requirements that were established prior to the implementation. Different levels of ease-of-circumvention were described in terms of system familiarity, system rigidity and authorization provisions (i.e., not organizational incentive mechanisms). The set of four composite cases was pilot tested for consistency and validity using groups of eight managers per case. Standard quantitative manipulation checks, as described in the full experiment, were run and qualitative feedback gathered suggesting significant distinctions among the cases along the factors of interest to our study.

Four versions of a survey, each including one of the manipulation cases, were administered to a set of managers enrolled in part-time and evening MBA courses at a business school ranked among the top-20 by The Financial Times and US News. Those subjects had a minimum 2 years' management experience. With the exception of the case summaries, the surveys were identical across all sample subjects (See Appendix A). Alternating the distribution of these distinct case questionnaires within recognizable subpopulations (e.g., male versus female) helped further reduce the risk of endogenous response biases among the four manipulations.

Prior to reading the case write-up, subjects were instructed to complete a set of questions aimed at eliciting standard control data. Respondents were also prompted for details regarding recent employment. Only after respondents provided their background information and read through their specific condition's case summary (i.e., after being conditioned by the experimental manipulation) were they given the opportunity to answer items relating to their intended shortand long-term reactions to the condition. At that point in the experiment, subjects were also given the opportunity to describe their perceptions of misfit and ease of circumvention. This data was collected primarily for use in standard checks to the interpretability of the manipulations.

The literature on task-technology fit provides general guidance in terms of assessing the degree of misfit perceived between imposed protocols and operational processes (Goodhue, 1998; Goodhue and Thompson, 1995). We adapt these scales to our interests in this study. Items include reverse-coded questions such as "To what extent do you believe the resource planning system protocols will match the level of information detail required for the established processing tasks?" and forward-coded items such as "To what extent do you believe the resource planning system protocols will not provide sufficient flexibility in dealing with the established processing tasks?" (See Appendix A for additional items). The mix of reverse- and forward-coded items helps guard against the risk of common internal response biases such as halo effects and acquiescence (Nunnally, 1978; Churchill, 1979). 
In developing an index designed to assess the interpretability of the ease-of-circumvention treatment, we drew on the established related literature on the "ease of system use" construct (Goodhue and Thompson, 1995; Somers et al., 2003). These items also mimic the scale items used to depict general perceptions of behavioral control in technology acceptance studies (Ajzen, 1991; Davis, 1989; Davis et al., 1989). Both forward and reverse-coded questions were again used to avoid response biases.

Since our main hypotheses deal with the intent of users to pursue circumvention options both immediately following go-live and in the long-term, we developed two separate scales for circumvention intent. These scales drew upon similar scales used in the literature on behavioral intent and applications of the theory of reasoned action (e.g., Fishbein and Ajzen, 1975; Gentry and Calantone, 2002). Items on this scale of short-term reactions included statements such as "As soon as the system goes live, I would be likely to adopt the type of processing activities supported by the system in place of my previous activities" (reverse-coded) and "As soon as the system goes live, I would be likely to attempt to make use of alternate procedures not directly supported by the system." Items used in the long-term intent scale were designed to mimic short-term items and were similarly coded.

\section{Analysis and results}

Out of the 426 managers solicited to voluntarily complete the survey experiment, 335 (79\%) provided complete responses. Of this group, $74 \%$ were male, $87 \%$ were born in the U.S., and English was the first language of $94 \%$. A comparison of the demographics of respondents and non-respondents showed no significant differences among responses, suggesting the absence of significant non-response bias in our sample.

\subsection{Manipulation checks and descriptive statistics}

To ensure that the manipulations provided appropriate contrasts between low and high levels of misfit as well as between low and high levels of perceived ease-of-circumvention 'environments', the levels of misfit and ease-of-circumvention (EOC) reported by respondents in each condition were compared. On the whole, given the presence of reverse coded items, the reliability of both misfit and ease-of-circumvention scales were adequate $(\alpha=0.832$ and 0.819 , respectively). Results from $t$-tests indicated that the means of perceived misfit reported for the high $(5.25$, S.D. $=0.71)$ and low $(2.77$, S.D. $=0.68)$ conditions were significantly different $(p<$ $0.001)$ and in the anticipated direction. Comparisons of average perceived levels ease-of-circumvention among the low $(2.85$, S.D. $=0.81)$ and high EOC $(5.36$, S.D. $=0.77)$ 
treatments showed similar results $(p<0.001)$. These results provide evidence of the discriminating capabilities of the treatments in the experimental manipulation, and thus justify the use of the treatments as a meaningful framework for comparing managerial intentions regarding use and circumvention tactics to be analyzed.

Descriptive statistics for perceived misfit, ease-of-circumvention, and short- and long-term intentions were calculated for each of the four controlled-manipulation cases. These are outlined in Table 2.

Although correlations between treatment perceptions and treatment reactions could be provided, their value to understanding the current research would be limited, since other perceptual measures are principally designed to validate the effectiveness of the experimental manipulation. It is more important to note that although there was a significant positive correlation between short-term and long-term intentions ( $p=0.417, p<0.01)$, no such significant relationships were detected within each of the four treatment groups. This suggests that these reactions were not highly subject to halo effects, but rather that variations in these responses were largely driven by the controlled independent treatments. Furthermore, since the treatments themselves seemed to provide compound impacts on the managerial intentions, several tests of combined treatment effects were warranted.

\subsection{Combined-effect tests}

We utilize two approaches to formally test our hypotheses. The first is a series of $t$-tests serving as planned comparisons of our sample subpopulations. The benefit of such an approach is in the ability to use controlled independent data (i.e., the experimental conditions) as a foundation for comparing subject responses, thus avoiding risks of common-source bias in the analysis. The use of planned $t$-tests has proven useful in discriminating controlled treatment effects in recent research into behavior in operational task studies (Schultz et al., 2003).

In this case, a check on the equality of sample variances is not only standard but also critical in illustrating the corollary hypothesis, Hypothesis 1b. Both Leven's test and Shoemaker's adjusted test for differences in sample variance suggest significantly greater variation in intent $(p$ $<0.005$ ) when EOC is high (Shoemaker, 2003). With such significant differences in variation we take a more conservative approach to testing for mean differences using Satterthwaite's adjusted $t$-test throughout the subsequent analyses (Armitage and Berry, 1994). For the sample as a whole, short-term intentions to circumvent tend to be greater when ease-of-circumvention $(E O C)$ is greater $(p<0.005)$. These differences are also clearly observable in both low and high misfit conditions tested separately ( $p<0.01$ and $p<0.001$, respectively). Variance differences 
were also somewhat apparent across these levels of EOC for both low and high misfit conditions $(p<0.10$ and $p<0.001$, respectively). The results of these planned comparisons provide initial support for Hypotheses $1 \mathrm{a}$ and $1 \mathrm{~b}$. Similar results with regard to average levels of intent, although not formally hypothesized, are observed related to long-term intention to circumvent $(p$ $<0.005$ and $p<0.05$, for low and high misfit respectively).

Planned comparisons for Hypotheses $2 a$ and $2 b$ are specifically aimed at observations based on the high misfit treatment. Under this condition no significant differences in mean and variance between short- and long-term intentions are observed for the high EOC treatment. However a significant difference ( $p<0.001$ in mean; $p<0.001$ in variance) between long and short-term intentions is observed at the low EOC level. Furthermore, under high misfit conditions, the mathematical difference between long-term and short-term intentions is significantly greater at the low EOC treatment than at the high EOC treatment $(p<0.01)$, as is the difference in variation $(p<0.001)$. Taken as a whole, the results of these planned comparisons support our hypotheses relating to the role of contingency on delayed effects (Hypotheses $2 a$ and $2 b$ ) and help justify the explanation of differences described in the Tristen and TECH cases.

To provide a still richer view of these contingencies a second approach to data analysis is also employed. This approach utilizes GLS to allow for the incorporation of demographic data and statistical evaluations of the impact of a binary interaction term (misfit $\times$ ease-of-circumvention) across both time frames. In the analysis we focus on the incremental $R^{2}$ change on the inclusion of potential interaction effects, beyond the individual primary effects of the two main independent variables, misfit and ease-of-circumvention. Results of these regression models are in Table 3.

The results presented in Table 3 illustrate several interesting points. First, in the presence of the interaction term (misfit $\times$ ease-of-circumvention), misfit seems to have a much greater impact on long-term intentions to circumvent than it does on short-term intentions. Second, the direct effect of ease-of-circumvention is significant yet appears to be nearly equivalent for both the short-term and long-term intentions. No significant differences between EOC coefficients in the two models were detected. In addition, the role of the interaction term seems to be relevant only with regard to short-term intentions. Specifically, while misfit demonstrates a larger impact on long-term intentions to circumvent systems, its effect on short-term reactions is moderated by the ease at which circumvention is available. The overall implication of this interaction effect is that short-term reactions to system changes will tend to be much more subdued than long-term reactions when misfit is high, unless ease-of-circumvention is also high.

In order to help further corroborate such effects we conducted analogous regressions 
using the subjective assessments of perceived misfit and perceived ease-of-circumvention reported by our subjects. Although in-line with the actual controlled treatments, as our manipulation checks suggest, it is possible that these reports of perceived contexts could be tapping into factors beyond those of the treatments and hence might yield somewhat different results. The results of these regressions however, as depicted in Table 4, show no major distinctions from the general results described by our controlled treatments, both corroborating the effects described as well as once again supporting the cleanness of the experimental treatments.

We note that in low misfit scenarios (left side of Fig. 6), there is little significant difference between short- and long-term intentions to circumvent, or in the variation of such intentions. This is true for both low and high EOC conditions. In high misfit scenarios (right side of Fig. 6), shortversus long-term differences in intention to circumvent are more dramatic. Most of these changes relate to temporal shifts in intentions where EOC is low. This shift in intentions is analogous to the conceptual shift originally depicted in the lower right cell of Fig. 3 and seems in line with our contingency and VIE related theoretical framework.

\subsection{Magnitude of intentions: post-hoc investigation}

One striking observation rising from our analysis was the scale of circumvention intention even among those subject to the low misfit treatment. While we had anticipated that individuals disdain changes of any kind in a work environment, regardless of perceived misfit, we had not expected such disdain to be so prominent in our laboratory study. For that reason, we re-examined our case data to surface other notable issues that might be incorporated in a post-hoc analysis of our laboratory observations. One particular theme seemed worthy of further consideration, namely the post-deployment absence of individuals at Tristen experienced with the resource and process interdependencies outlined through the ES implementation. In contrast, TECH deployed "Super-Users" with extensive appreciation for the interdependencies of the ES across the enterprise.

Recent literature draws on information processing theory in order to better understand perceptions of ERP usefulness. This work has shown that those with supervisory experience tend to be more in tune with the interdependencies of work settings (Bachrach et al., 2006). These individuals also tend to be more open to new technologies (e.g., ERP) that hold the potential for facilitating interdependent work. Such experience is seen to heighten the valuation of ERP systems and, we speculate, also limits tendencies to act in ways that reduce such value or increase variation in system use. Accordingly, in the context of our study, we would expect 
that individuals with higher levels of supervisory experience would be less likely to voice an intention to circumvent regardless of time-frame (i.e., short- or long-term). Similarly we would expect that those with greater supervisory experience would exhibit less variation in their stated intentions to deviate, than those with less experience.

In order to test for these effects and to, in part, attempt to confirm recent findings of the importance of supervisory experience on perceptions of ERP systems, we performed additional regression analyses incorporating past supervisory experience as an additional experiential control. To keep the scope of the post-hoc investigation manageable, we focus here on the time-frame of greatest interest to this study-the long-term over which learning mechanisms might reveal new opportunities for circumvention. Table 5, illustrates how the inclusion of this experience significantly increases observed $R^{2}$ without appreciably altering the effects of other independent variables in the model.

We also conducted a post-hoc, split-sample analysis comparing sub-populations of our sample for which past supervisory experience was low (three individuals supervised or less) or relatively high (20 or more individuals supervised). In all treatment settings of our split-sample analysis, our data showed that those with greater past supervisory experience were less likely to indicate an intention to pursue long-term circumvention. The observed effects are graphically illustrated in Fig. 7.

Beyond further illuminating the nuances related to long-term ERP circumvention, these findings provide prescriptive insights for managers seeking to reduce variation in use by lower level personnel. Specifically, employee exposure to supervisory or other opportunities which reveal organizational interdependencies may yield beneficial increases in the stability of new system usage.

\subsection{Experimental limitations}

An important issue to note with regard to these results is that the treatment conditions used in our experiment are, by design, binary-presenting only contexts with high or low misfit and ease of circumvention. In many practical settings, levels of misfit and ease of circumvention may be more naturally characterized as a matter of degree. Auxiliary analyses conducted concurrently with this research provided a partial account for such continuously scaled characterizations. The results were consistent with those involving the binary controls. Regardless, there may still be some concern about single-source biases and halo effects associated with such models. An objective, continuous assessment of both misfit and ease-of-circumvention would ultimately have been preferable. More sophisticated laboratory 
designs involving multiple levels of controlled misfit and ease-of-circumvention levels could provide one solution for future validation. Perhaps more meaningful however would be true in-situ action-research using independent and objective sources for both continuous independent and dependent variables. Provided sufficient account of other extraneous effects, the findings of such work could be extremely compelling.

We also note that our experimental findings focus on intent, rather than actual outcomes. As stated earlier, this focus was the result of our effort to isolate the independent factors under investigation, thus making the use of action-research impractical for the targeted sample size required for this first level of phenomenological validation. Because of this our work remains silent on the issue of whether this intent will actually result in realization of short-term or long-term circumventions, or even in their attempt. We hasten to add, however, that measures of intent have been successfully used related studies to successfully evaluate behavioral issues surrounding IT implementation (Ajzen, 1991; Taylor and Todd, 1995; Taylor and Tucker, 1989).

\section{Discussion}

Organizations can be characterized as collectives of processes, dependent on the individual agents that carry them out and the rule-structures that constrain those individuals. Despite the presence of constraining protocols, individuals may have a variety of alternatives available to them for carrying out processes. Furthermore, the scope of alternatives may change over time. In our view, the array of alternatives, coupled with differences in individual characteristics, represents a natural source of observable process variation in organizations. Although certain rule-structures may limit alternatives in the short-term, hence suppressing process variation, the tendency for individuals to attempt to circumvent these structures in the long-term may be heightened by contingent factors such as perceived misfit and ease of circumvention. Our theoretical framework is thus representative of a valence-instrumentality-expectation perspective of task-technology fit and resultant operational dynamics. We specifically apply our framework here to illustrate the phenomenon of growth in process variation over time.

\subsection{Contribution}

Our theoretical framework is unique in its emphasis on evolving dynamics and contingencies upon which trends in process variation depend. Distinct from a lockstep phase-based view of adaptation and acceptance, our framework requires a willingness to view these "phases" as interwoven. Our framework further suggests that it may be misleading to view 
any single time-reference as indicative of stable appropriation when appropriation equilibria both evolve over time and may not be accurately described as "faithful" (i.e., may be largely an artifact of circumventions that become norms or portfolios of practice). Using the contemporary rule-structure context of newly imposed ERP protocols, we examine the interplay of system misfit and ease-of-circumvention over time as they relate to the emergence of variation in use.

Evidence in support of our contingency view of process variation is drawn from both case and experimental study, a combination that is particularly well-suited, yet rarely applied despite calls for greater use of process-oriented methods in research (c.f. Choi et al., 2001; Poole and DeSanctis, 2004). Our experimental findings reveal that for contexts in which strong perceptions of misfit exist, the intentions of managerial users depend both upon the apparent availability of circumventions and their timing. Where circumventions are straightforward and available (i.e., High-EOC contexts), misfit perceiving managers signal strong intention to circumvent imposed protocols in the short-term as well as in the long-term. Still more interesting are managerial reports of persistent intention in contexts in which workarounds are less immediately obvious (i.e., Low-EOC contexts). Under such conditions managers simultaneously signal an early willingness to comply with the system as deployed, and a continued intent to seek circumvention in the long-term. These findings mimic our case examinations wherein managers attributed changes in process variation to individual perceptions of protocol misfit and the availability of workarounds. While, admittedly, "intentions" are not strictly equivalent to "actions," it is not improbable that the temporal dynamics associated with intentions to circumvent may at least partially translate into undesirable, often unanticipated, anomalies observable through operating data, as suggested by the Tristen case.

\subsection{Future work}

From a general "measurements" perspective, one must acknowledge that there are many possible operational metrics upon which firms can focus (c.f. Melnyk, 1999). The findings of this study may, therefore, not be relevant to all firms. Although appropriate for the firms and experimental context used here, we would stress caution in attempting to perform similar studies at more generalized firm-level of analysis (e.g., surveys across firms that might have very different operational missions). Managers at different firms are likely to have different views of what is important.

Regardless of measure, we note that studies employing snapshot approaches to performance assessment face a risk of encountering different stages of resistance and adaptation to innovations. These studies have little hope of accounting for variation due to 
sustained perceptions of misfit coupled with learning mechanisms if they do not account for the amount of time new systems have been in place. Studies that account for time-passed but not for the rates at which learning and adaptation occur face similar difficulties in interpretability. Only studies that capture temporal dynamics, based on at least some limited level of longitudinal data, have a real chance of providing new insights into the linkages between technology and sustainable performance.

Equipped with rich longitudinal data on multiple system-change environments, theoretical understanding of these environments may reach beyond the tri-part contingency framework posited here. Although implicit to the development of evolving circumvention tactics, joint examinations of learning, misfit, and non-prescribed action over time could provide valuable material for the construction of truly rich models of change and adaptation. Mental models of "what fits" may change over time, based on the ease by which alternatives can be pursued (i.e., the perception of misfit may diminish if circumvention is thwarted over a long enough period). On the other hand, continued discomfort with imposed protocols among a small number of individuals may be voiced socially, spreading the perception of misfit among those who initially found the protocols to be acceptable.

It is also conceivable that, given enough time, individuals faced with even very different levels of ease of circumvention and perceived misfit may approach an asymptotic level of variation in use - some equilibrium at which dissatisfaction with existing options is balanced by incremental difficulty in pursuing alternative options. Transitions between semi-stable states limited by actionable knowledge (i.e., means of circumvention), dynamic states in which alternative actions are tested and evolve, and later restabilization (e.g., when discomfort has largely abated and additional options for circumvention do not warrant pursuit) are akin to the structure-agency iterations suggested in recent organizational theory (Boudreau and Robey, 2005; Leanna and Barry, 2000). They are further representative of the temporal dynamics that Meyer et al. (2005) suggest more realistically characterize many organizations. Tracking such dynamics through time could allow predictive analyses and, if these events are tied to identifiable antecedents, allow the anticipation of such events in settings where system-change has yet to take place (with obvious benefits).

We also note that that "misuse" of a system relative to the "use" initially intended by management is not necessarily a bad thing. Several authors suggest that in some cases such unintended approaches to use may be beneficial to both individuals and the organization (Rogers, 1983; Narayanan, 2001). However, in some cases, where for example control and variation reduction is the explicit objective, actions that appear to benefit the user may be 
extremely disruptive to the "global" operational goals of the firm.

Related to operational practice, the findings of this study imply a need for strong communication during system deployment, and for continuous monitoring beyond go-live. Given that learning comes from experience, sustained discomfort with imposed IT structures may lead to severe misinterpretations of point estimates of performance by practitioners and researchers alike (it most likely already has in many cases). One tactic to reduce the risk of delayed circumvention may be an attempt to eliminate all "true" misfit between operational processes and system protocols. Of course, such efforts may be time-and cost-prohibitive.

An alternative tactic may be the attempt to manage organizational perceptions of misfit. This does not necessarily imply that managers must concede to local whim with regard to tailoring the system to the local context. As in the case of $\mathrm{TECH}$, corporate interests may not be willing to acquiesce to local objections. Rather, they may choose to continue to broadcast the local benefits of the protocols instantiated by the system. This may involve formal shifts in local performance objectives and compensation structures as alluded to by researchers such as Griffith et al. (1999). Some emphasis on increasing the difficulty of circumvention may be warranted given the construct's significant correlation with intention, though we would recommend further investigation into the materiality of that difference. More suggestive is the potential for further training in "faithful" use, provided that it is designed to match operational and strategic goals. As suggested by the results of our post-hoc examination, encouraging supervisory opportunities for individuals may enhance appreciation of interdependencies and promote ES benefits. Such appreciation could discourage individual attempts at circumvention as well as reduce the likelihood that such circumvention would drive excessive and unanticipated levels of process variability into the operations.

\section{Notes}

a Emory University, 1300 Clifton Road, Atlanta, GA 30322-2710, United States

b Marquette University, P.O. Box 1881, Milwaukee, WI 53201, United States

* Corresponding author. Tel.: +1 4047277138.

- E-mail addresses: elliot_bendoly@bus.emory.edu (E. Bendoly), mark.cotteleer@marquette.edu (M.J. Cotteleer).

1 Tel.: +14142885493.

\section{References}


Ajzen, I., 1991. The theory of planned behavior. Organizational Behavior and Human Decision Processes 50, 179-211.

Amoako-Gyampah, K., Salam, A.F., 2004. An extension of the technology acceptance model in an ERP implementation environment. Information and Management 41, 731-745.

Armitage, P., Berry, G., 1994. Statistical Methods in Medical Research, third ed. Blackwell, Oxford.

Bachrach, D.G., Powell, B., Bendoly, E., Richey, G., 2006. Organizational citizenship behavior and performance evaluations: Exploring the impact of task interdependence. Journal of Applied Psychology 91 (1), 120-137.

Bachrach, D.G., Bendoly, E., Podsakoff, P.M., 2001. Attributions of the 'causes' of performance as an alternative explanation of the organizational citizenship behavior/organizational performance relationship. Journal of Applied Psychology 86 (6), 1285-1293.

Barki, H., Pinsonneault, A., 2005. A model of organizational integration, implementation effort and performance. Organization Science 16 (2), 165-179.

Beaudry, A., Pinsonneault, A., 2005. Understanding user responses to information technology: a coping model of user adaptation. MIS Quarterly 29 (3), 493-524.

Bendoly, E., 2001. ERP systems and supply chain technologies: a caveat for B2B e-procurement. FedEx White Paper Series, White-paper 28-36.

Bendoly, E., Jacobs, R., 2004. ERP process and operations task alignment: performance insights at the order processing level. International Journal of Operations and Production Management 24 (1), 99-117.

Bendoly, E., Jacobs, R.F., 2005. Strategic ERP Extension and Use. Stanford University Press, Santa Clara, CA

Bendoly, E., Kaefer, F., 2004. Business technology complementarities: impact of the presence and timing of ERP on B2B e-commerce technology efficiencies. Omega 32 (5), 395-405.

Bendoly, E., Swink, M., 2007. Moderating effects of information access on project management behavior, performance and perceptions. Journal of Operations Management 25 (3), $31-54$

Boudreau, M., Robey, D., 2005. Enacting integrated information technology: a human agency perspective. Organization Science 16 (1), 3-18.

Boyce, T., 2001. Sometimes we must break the rules to maintain behavior change: a response to Baer, Fleming, Malott, and McSween and Matthews. Journal of Organizational Behavior Management 21 (1), 103-112.

Brynjolfsson, E., Hitt, L., 1996. Paradox lost? Firm-level evidence on the returns to information 
systems spending. Management Science 42 (4), 541-558.

Brynjolfsson, E., Hitt, L., 2003. Computing productivity: firm-level evidence. The Review of Economics and Statistics 85 (4), 793-808.

Brynjolfsson, E., Hitt, L., Yang, S., Baily, M., Hall, R., 2002. Intangible assets: computers and organizational capital. Brookings Papers on Economic Activity 1, 137-198.

Chau, P.Y.K., Hu, P.J., 2001. Information technology acceptance by individual professionals: a model comparison approach. Decision Sciences 32 (4), 699-719.

Choi, T.Y., Dooley, K.J., Rungtusanatham, M., 2001. Supply networks and complex adaptive systems: control versus emergence. Journal of Operations Management 19, 351-366.

Churchill, G.A., 1979. A paradigm for developing better measures of marketing constructs. Journal of Marketing Research 16, 64-73.

Cotteleer, M.J., 2006. An empirical study of operational performance parity following enterprise system deployment. Production and Operations Management 15 (1), 74-88.

Cotteleer, M.J., Bendoly, E., in press. Order lead-time improvement following enterprise-IT implementation: An empirical study. MIS Quarterly.

Davenport, T.H., 1998. Putting the enterprise into the enterprise system. Harvard Business Review 76 (4), 121-131.

Davenport, T.H., 2000. Mission Critical. Harvard Business School Press, Boston.

Davis, F.D., 1989. Perceived usefulness, perceived ease of use and user acceptance of information technology. MIS Quarterly 13, 319-340.

Davis, F.D., Bagozzi, R.P., Warshaw, P.R., 1989. User acceptance of computer technology. Management Science 35, 982-1003.

DeSanctis, G., Poole, M.S., 1994. Capturing the complexity in advanced technology use: adaptive structuration theory. Organization Science 5, 94-118.

Dishaw, M.T., Strong, D.M., 1999. Extending the technology acceptance model with task-technology fit constructs. Information and Management 36 (1), 9-13.

Dougherty, D., 2001. Re-imagining the differentiation and integration of work for sustained product innovation. Organization Science 12 (5), 612-631.

EI Sawy, O.A., Majchrzak, A., 2004. Critical issues in research on real-time knowledge management in enterprises. Journal of Knowledge Management 8 (4), 21-37.

Festinger, L., 1957. The Theory of Cognitive Dissonance. Stanford University Press, Stanford, CA.

Fishbein, M., Ajzen, I.,1975. Belief, Attitude, Intention and Behavior: An Introduction to Theory and Research. Addison-Wesley, Reading, MA. 
Frei, F.X., Kalakota, R., Leone, A.J., Marx, L.M., 1999. Process variation as a determinant of bank performance: evidence from the retail banking study. Management Science 45 (9), 1210-1220.

Garvin, D.A., 1998. The processes of organization and management. Sloan Management Review 39 (4), 33-50.

Gentry, L., Calantone, R., 2002. A comparison of three models to explain shop-bot use on the web. Psychology and Marketing 19 (11), 945-956.

George, E., 2003. External solutions and internal problems: the effects of employment externalization on internal workers' attitudes. Organization Science 14 (4), 386-402.

Goodhue, D.L., 1995. Understanding user evaluations of information systems. Management Science 41 (12), 1827-1844.

Goodhue, D.L., 1998. Development and measurement validity of a task-technology fit instrument for user evaluations of information systems. Decision Sciences 29 (1), 105-138.

Goodhue, D.L., Thompson, R.L., 1995. Task-technology fit and individual performance. MIS Quarterly 19 (2), 213-236.

Gopal, A., Bostrom, R.P., Chin, W.W., 1992. Applying adaptive structuration theory to investigate the process of group support systems use. Journal of Management Information Systems 9 (3), 45-69.

Griffith, T.L., Tansik, D.A., Leman, B., 2002. Negotiating technology implementation: an empirical investigation of a website introduction. Group Decision and Negotiation 11, $1-22$.

Griffith, T.L., Zammuto, R.F., Aiman-Smith, L., 1999. Why new technologies fail: Overcoming the invisibility of implementation. Industrial Management 41, 29-34.

Jacobs, R., Bendoly, E., 2003. Enterprise resource planning: developments and directions for operations management research. European Journal of Operational Research 146 (2), 5-12.

Leanna, C.R., Barry, R., 2000. Stability and change as simultaneous experiences in organizational life. Academy of Management Review 25, 753-759.

Lee, Z., Lee, J., 2000. An ERP implementation case study from a knowledge transfer perspective. Journal of Information Technology 15, 281-288.

Legris, P., Ingham, J., Collerette, P., 2003. Why do people use information technology? A critical review of the technology acceptance model. Information and Management 40 (3), 191-204.

Light, B., Holland, C., Wills, K., 2001. ERP and best of breed: a comparative analysis. Business 
Process Management Journal 7 (3), 216-224.

Mabert, V., Soni, A., Venkataramanan, M.A., 2003. Enterprise resource planning: Managing the implementation process. European Journal of Operational Research 146 (2), 302-322.

Mahmood, M.A., Hall, L., Swanberg, D.L., 2001. Factors affecting information technology usage: a meta-analysis of the empirical literature. Journal of Organizational Computing and Electronic Commerce 11 (2), 107-130.

Mathieson, K., 1991. Predicting user intention: comparing the technology acceptance model with theory of planned behavior. Information System Research 2 (3), 173-191.

Mayes, B.T., 1978. Incorporating time-lag effects into the expectancy model of motivation: a reformulation of the model. Academy of Management Review 3 (2), 374-380.

McAfee, A., 2002. The impact of enterprise technology adoption on operational performance: an empirical investigation. Production and Operations Management 11 (1), 33-53.

Melnyk, S.A., 1999. Measurements, Metrics and the Value-Driven Operations Management System. Lionheart Publications, Atlanta, GA.

Meyer, A.D., Gaba, V., Colwell, K.A., 2005. Organizing far from equilibrium: non-linear change in organizational fields. Organization Science 16 (5), 456-473.

Narayanan, V.K., 2001. Managing Technology and Innovation for Competitive Advantage. Prentice Hall, Englewood Cliffs.

Nunnally, J.C., 1978. Psychometric Theory, 2nd ed. McGraw-Hill, New York.

Oreg, S., 2003. Resistance to change: Developing an individual differences measure. Journal of Applied Psychology 88 (4), 680-697.

Orlikowski, W.J., 2000. Using technology and constituting structures: a practice lens for studying technology in organizations. Organization Science 11 (4), 404-428.

Poole, M.S., DeSanctis, G., 2004. Structuration theory in information systems research: methods and controversies. In: Whitman, M.E., Moszerynski's, A.B. (Eds.), The Handbook of Information Systems Research. Idea Group Pub, New York, pp. 206-248.

Rabinovich, E., Dresner, M., Evers, P., 2003. Assessing the effects of operational processes and information systems on inventory performance. Journal of Operations Management 21 (1), 63-80.

Razi, M.A., Tarn, J.M., 2003. An applied model for improving inventory management in ERP systems. Logistics Information Management 16 (2), 114-124.

Robbins, S.P., 1986. Organizational Behavior: Concepts, Controversies and Applications. Prentice-Hall, Englewood Cliffs, NJ.

Robey, D., Ross, J.W., Boudreau, M., 2002. Learning to implement enterprise systems: an 
exploratory study of the dialectics of change. Journal of Management Information Systems 19 (1), 17-46.

Rogers, E.M., 1983. Diffusion of Innovations, third ed. The Free Press, New York.

Saxberg, B.O., Slocum, W., 1968. The management of scientific manpower. Management Science 14 (8), 473-489.

Scheer, A.W., Habermann, F., 2000. Making ERP a success. Communications of the ACM 43 (4), 57-61.

Schultz, K.L., McClain, J.O., Thomas, L.J., 2003. Overcoming the dark side of worker flexibility. Journal of Operations Management 21 (1), 81.

Schultz, K.L., Juran, D.C., Boudreau, J.W., 1999. The effects of low inventory on the development of productivity norms. Management Science 45 (12), 1664-1678.

Schultze, U., Orlikowski, W.J., 2004. A practice perspective on technology-mediated network relations: the use of Internet-based self-serve technologies. Information Systems Research 15, 87-106.

Seely Brown, J., Hagel, J., 2004. Flexibile IT, better strategy. McKinsey Quarterly 4.

Shoemaker, L.H., 2003. Fixing the $\mathrm{F}$ test for equal variances. The American Statistician 57 (2), 105-114.

Soh, C., Sia, S.K., Boh, W.F., Tang, M., 2003. Misalignment in ERP implementation: a dialectic perspective. International Journal of Human-Computer Interaction 18 (1), 81-100.

Soh, C., Sia, S.K., Tay-Yap, J., 2000. Cultural fits and misfits: is ERP a universal solution? Communications of the ACM 43 (4), 48-51.

Somers, T.M., Nelson, K., Karimi, J., 2003. Confirmatory factor analysis of end-user computing satisfaction instrument: replication within an ERP domain. Decision Sciences 34 (3), 595-621.

Stein, E.W., Vandenbosch, B., 1996. Organizational learning during advanced system development: opportunities and obstacles. Journal of Management Information Systems $13(2), 115-136$.

Szajna, B., Scamell, R.W., 1993. The effects of information system user expectations on their performance and perceptions. MIS Quarterly 17 (4), 493-516.

Stratman, J.K., Roth, A.V., 2002. Enterprise resource planning (ERP) competence constructs: Two-stage multi-item scale development and validation. Decision Sciences 33 (4), 601-628.

Taylor, S., Todd, P.A., 1995. Understanding information technology usage: a test of competing models. Information Systems Research 6 (2), 144-176. 
Taylor, J.R., Tucker, C.C., 1989. Reducing data processing costs through centralization. MIS Quarterly 13 (4), 487-499.

Trott, P., Hoecht, A., 2004. Enterprise resource planning and the price of efficiency: The trade off between business efficiency and the innovative capacity of firms. Technology Analysis \& Strategic Management 16 (3), 367-379.

Tyre, M.J., Orlikowski, W.J., 1994. Windows of opportunity: temporal patterns of technological adaptation in organizations. Organization Science 5 (1), 98-118.

Tyre, M.J., von Hippel, E., 1997. The situated nature of adaptive learning in organizations. Organization Science 8 (1), 71-83.

Umanath, N.S., 2003. The concept of contingency beyond "It depends": illustrations from IS research stream. Information and Management 40 (6), 551-562.

Van Stijn, E., Wensley, A., 2001. Organizational memory and the completeness of process modeling in ERP systems. Business Process Management 7 (3), 181-194.

Venkatesh, V., 1999. Creation of favorable user perceptions: exploring the role of intrinsic motivation. MIS Quarterly 23 (2), 239-260.

Venkatesh, V., Davis, F., 2000. A theoretical extension of the technology acceptance model: four longitudinal field studies. Management Science 46 (2), 186-194.

Venkatesh, V., Speier, C., Morris, M.G., 2002. User acceptance enablers in individual decision making about technology: Toward an integrated model. Decision Sciences 33 (2), 297-317.

Venkatesh, V., Morris, M.G., Davis, G.B., Davis, F.D., 2003. User acceptance of information technology: towards a unified view. MIS Quarterly 27 (3), 425-477.

Vroom, V.H., 1964. Work and Motivation. Wiley, New York.

Weick, K.E., 1998. Improvisation as a mindset for organizational analysis. Organization Science 9, 543-555.

Weill, P., Olson, M.H., 1989. An assessment of the contingency theory of management information systems. Journal of Management Information Systems 6 (1), 59-85.

Weiss, H.M., Nicholas, J.P., Daus, C.S., 1999. An examination of the joint effects of affective experience and job beliefs on job satisfaction and variations in affective experiences over time. Organizational Behavior and Human Decision Processes 78 (1), 1-24.

Yin, R.K., 1994. Case Study Research: Design and Methods. Sage Publications, London, UK.

Zammuto, R.F., O'Connor, E.J., 1992. Gaining advanced manufacturing technologies' benefits: the roles of organization design and culture. Academy of Management Review 17 (4), 701-728. 


\section{Appendix A}

\section{Selected Pre-Condition Demographic Data}
a) Age
b) Gender
c) Number of years of full-time work experience

d) Number of years of full-time work experience

e) Industry in which you are currently / were most recently employed

f) Years with current organization

g) Current / most recent job title

[All above responses are open scaled]

h) Given the opportunity to circumvent a new procedure built into an information system at work, how likely would be to attempt to do so (i.e., avoid that procedure) in order to limit changes in the way your job is done? [Likert-type item: Never $=1$, Highly Likely $=7$ ]

\section{Case Conditions}

[Combinations of the "High" and "Low" misfit and ease-of-circumvention conditions outlined below were used to construct the four case conditions in the experiment; Terms in brackets not provided on actual questionnaire]

\section{[Paragraphs 1 and 2 for the "Low Misfit" conditions]}

"A firm that develops and manufactures electronic monitoring devices is about to go-live with a new enterprise resource planning system. Over the years the firm has established a reputation as a low cost provider to the needs of a domestic clientele base that spans only a few select industries. The limned number of stable product options available drives both a make-to-stock philosophy at the firm as well as relatively basic and homogeneous order types. As a result the firm's ability to depend on standard orders from its clientele has allowed it to maintain a low cost structure in line with its competitive strategy. If such basic standardization is not matched by effective data processing capabilities, the firm risks losing business opportunities and market confidence. A lack of distinction among the types of clientele encountered by their multiple domestic business units only emphasizes the appropriateness of support for such integrated standardization in maintaining the firm's scale economies.

Prior to go-live, the order processing procedures at the firm were considered "mature", supported by a complex array of locally customized legacy systems. However, a lack of local language and formatting differences at the various regional divisions had suggested that certain levels of redundancy exist across these diverse systems, and had called to question the need for such independently designed and managed systems. It further cast doubt on whether these long-standing systems had in fact been providing comparable levels of operational support. The 
deployment of the new enterprise-wide system was seen by management as a means to diffuse these concerns. Furthermore, the system's use of a single data formatting mechanism is viewed a means to ensure information for similar orders across regional business units are in fact entered into identical data fields, to allow for comparability and simple data aggregation. Additionally, the actual order processing protocols supported by the new system are highly standardized to meet the needs of centralized order management practices. As a result the processing sequence used by order managers across all divisions can now follow a common set of steps, thus facilitating company-wide process analysis."

\section{[Paragraphs 1 and 2 for the "High Misfit" conditions]}

"A firm that develops and manufactures electronic monitoring devices is about to go-live with anew enterprise resource planning system. Over the years the firms has established a reputation for remaining flexible to the needs of an international clientele base that spans multiple industries. Make-to-order is the norm, giving rise to a range of heterogeneous and complex order types. As a result the firm's ability to customize orders to the diverse needs of its clientele has become a competitive factor driving its success. If such customization cannot be provided smoothly and without notable restriction, the firm risks losing business opportunities and market confidence. Differences in the types of clientele encountered by different regional business units makes the need for local customization even more critical as far as global competitiveness and the maintenance of the firm's scale economies is concerned.

Prior to go-live, the order processing procedures at the firm were considered "mature", supported by a complex array of locally customized legacy systems. In fact, local language and formatting differences at the various regional divisions has created translation needs that are not entirely met by the capabilities built into the new enterprise resource planning system. Higher-level management has not seen this as an major issue and has requisitioned an additional bolt-on application to help to account for this. Regardless the system's use of a single data formatting mechanism will require certain information for less common local orders to be entered ad-hoc into data fields not originally designed to store such data. Furthermore the actual order processing protocols supported by the new system are highly standardized to meet the needs of centralized order management practices. As a result the processing sequence used by order managers for certain locally specific orders that had previously been handled by separate legacy systems will now follow a sequence more common to the majority of standardized orders." 
[Paragraph 3 for the "Low Ease of Circumvention" conditions]

"All of these required formatting and sequencing changes associated with the transition to the new resource planning system have been fully described to the staff of order managers who will be the predominant users of the order-processing module, However, because of their roles, these managers have not been extensively trained with the data storage and access capabilities of the system, As a result, order information cannot be readily accessed, modified and or entered into the central database without following the steps outlined by system protocols. Attempts at system bypassing are not recommended by the system developers and in-house IT staff, since they argue the system protocols are designed to provide best practices and checks and balances to reduce order entry errors. Furthermore, formal authorization barriers have been built into the system to deter such unorthodox system use."

\section{[Paragraph 3 for the "High Ease of Circumvention" conditions]}

"All of these required formatting and sequencing changes associated with the transition to the new resource planning system have been fully described to the staff of order managers who will be the predominant users of the order-processing module. However, these managers have also been extensively trained with the data storage and access capabilities of the system. If necessary, order information can readily be accessed, modified and or entered directly into the central database without following the steps outlined by system protocols. Such system bypassing is not recommended by the system developers and in-house IT staff, since they argue the system protocols are designed to provide best practices and checks and balances to reduce order entry errors. Regardless, no formal authorization barriers deter such unorthodox system use."

\section{Post-Condition Survey Items}

[Terms in brackets not provided on actual questionnaire]

Imagine you are in the role of an order-processing manager at this firm. Please indicate the extent to which you agree with the following statements.

[Also item responses are Likert-type with $1=$ Strongly Disagree $\ldots 4=$ Neutral $\ldots 7=$ Strongly Agree]

[Extent of Misfit] 
To what extent do you believe the resource planning system protocols...

1.a)...will match the level of information detail required for the established order processing tasks (R)

1.b)...will be easy to select and access when appropriate for each type of order processing task to be dealt with $(R)$

1.c)...will not provide sufficient flexibility in dealing with the established order processing tasks

1.d)...will not provide sufficient authorization to quickly meet the needs of the order processing tasks dealt with

1.e)...will not require additional steps that are unrelated to the existing order processing tasks (R) 1.f)...will not provide comparable interpretability to the established terminology/formats used in processing

\section{[Ease-of-Circumvention]}

2.a) It will not be easy to learn how to work around the standard protocols of the resource planning system $(R)$

2.b) Once a tactic is developed, the resource planning system's built-in protocols will be easy to circumvent

2.c) Working around the resource planning system's standard protocols should not require a great deal of additional time and effort

2.d) Circumventing the "locked-in" requirements of system-use will be challenging (R)

\section{[Intent to Circumvent-Short-term (Immediate)]}

As soon as the system goes live, I would be likely to...

3.a)... adopt the type of processing activities supported by the system in place of my previous activities $(R)$

3.b)... change the order of my previous work to accommodate the processing sequence supported by the system $(R)$

3.c)... modify the type of information I focus on to match the data input requirements of the system (R)

As soon as the system goes live, I would be likely to attempt to...

3.d)... use alternate information sources and records external to the system to support processing

3.e)... bypass steps that are supported by the system but not part of previous operating procedures 
3.f)... make use of alternate procedures not directly supported by the system

\section{[Intent to Circumvent-Long-term]}

Knowing what I know of the firm's operations, I will be reluctant to permanently...

4.a)... adopt the type of processing activities supported by the system in place of my previous activities

4.b)... change the sequence of my previous work to accommodate the processing sequence supported by the system

4.c)... modify the type of information I focus on to match the data input requirements of the system

After the system is in place and I have begun to gain some familiarity with its functionality, I am likely to...

4.d)... avoid alternate information sources and records external to the system to support processing $(R)$

4.e)... ignore ways to bypass steps supported by the system but not part of previous operating procedures $(\mathrm{R})$

4.f)... pass up and disregard alternate procedures not directly supported by the system (R) 


\section{Appendix B}

\section{Table 1 Experimental framework and associated theoretized outcomes}

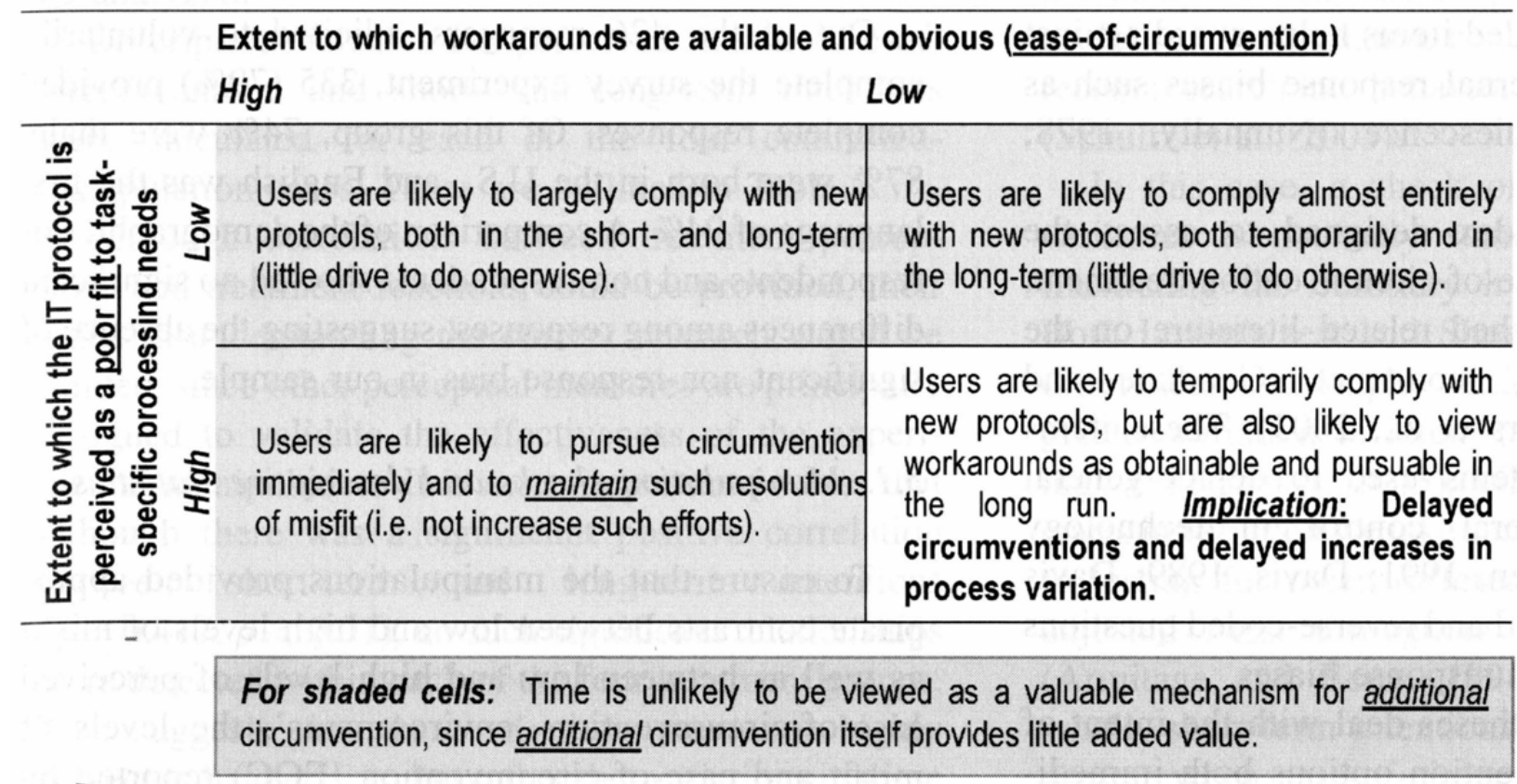

Table 2 Descriptives for perception-checks and outcomes at each treatment

\begin{tabular}{|c|c|c|c|c|}
\hline & Extent to which workarounds ar & re available & d obvious (ease-of-circumventio & \\
\hline & High & & Low & \\
\hline 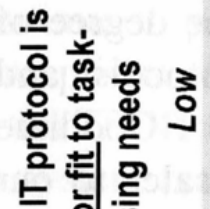 & $\begin{array}{l}\text { Perceived Misfit: } \\
\text { Perceived Ease-of-Circumvention: } \\
\text { Intent to Circumvent (Short-term): } \\
\text { Intent to Circumvent (Long-term): }\end{array}$ & $\begin{array}{l}2.78(0.65) \\
5.37(0.74) \\
3.31(0.64) \\
3.26(0.68)\end{array}$ & $\begin{array}{l}\text { Perceived Misfit: } \\
\text { Perceived Ease-of-Circumvention: } \\
\text { Intent to Circumvent (Short-term): } \\
\text { Intent to Circumvent (Long-term): }\end{array}$ & $\begin{array}{l}2.76(0.72) \\
2.82(0.79) \\
2.73(0.61) \\
2.68(0.63)\end{array}$ \\
\hline 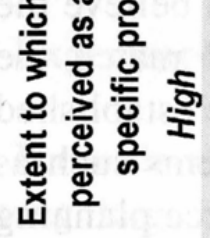 & $\begin{array}{l}\text { Perceived Misfit: } \\
\text { Perceived Ease-of-Circumvention: } \\
\text { Intent to Circumvent (Short-term): } \\
\text { Intent to Circumvent (Long-term): }\end{array}$ & $\begin{array}{l}5.22(0.73) \\
5.35(0.80) \\
4.78(0.83) \\
5.12(0.88)\end{array}$ & $\begin{array}{l}\text { Perceived Misfit: } \\
\text { Perceived Ease-of-Circumvention: } \\
\text { Intent to Circumvent (Short-term): } \\
\text { Intent to Circumvent (Long-term): }\end{array}$ & $\begin{array}{l}5.27(0.70) \\
2.88(0.85) \\
3.15(0.62) \\
4.67(0.99)\end{array}$ \\
\hline
\end{tabular}




\section{Table 3 GLS regressions of controlled treatments and interactions on circumvention forms}

\begin{tabular}{|c|c|c|c|c|c|}
\hline & \multicolumn{2}{|c|}{$\begin{array}{l}\text { DV: short-term } \\
\text { circumvention }\end{array}$} & \multicolumn{2}{|c|}{$\begin{array}{l}\text { DV: long-term } \\
\text { circumvention }\end{array}$} & \multirow[t]{2}{*}{ Test of coeff. diffs. } \\
\hline & $\beta$ & S.E. & $\beta$ & S.E. & \\
\hline \multicolumn{6}{|l|}{ Controls } \\
\hline Constant & $2.74^{* *}$ & 0.10 & $2.68^{* *}$ & 0.08 & n.s. \\
\hline Age & $0.03^{*}$ & 0.01 & 0.01 & 0.02 & $p<0.05$ \\
\hline Gender $($ male $=0$, female $=1)$ & -0.08 & 0.06 & $-0.09^{*}$ & 0.03 & n.s. \\
\hline \multicolumn{6}{|l|}{ Primary independents } \\
\hline Misfit $($ low $=0$, high $=1)$ & $0.41^{* *}$ & 0.10 & $1.99^{* *}$ & 0.13 & $p<0.01$ \\
\hline Ease-of-circumvention $($ low $=0$, high $=1)$ & $0.58^{* *}$ & 0.12 & $0.57^{* *}$ & 0.11 & n.s. \\
\hline \multicolumn{6}{|l|}{ Interaction term } \\
\hline Misfit $\times$ ease-of-circumvention & $1.05^{* *}$ & 0.19 & 0.04 & 1.63 & $p<0.01$ \\
\hline$\Delta R^{2}$ (on addition of interaction term) & $0.048^{* *}$ & & 0.004 & & \\
\hline Final $R^{2}$ & $0.453^{* *}$ & & $0.644^{* *}$ & & \\
\hline
\end{tabular}

Note. Since no other preliminary controls are significant in the regressions, either w.r.t. total model $R^{2}$ or factor coefficients, their numbers are excluded from this table. ${ }^{\star \star} p<0.01$, one-tailed, ${ }^{*} p<0.05$, one-tailed.

\section{Table 4 GLS regressions of perceptual measures and interactions on circumvention forms}

\begin{tabular}{|c|c|c|c|c|c|}
\hline & \multicolumn{2}{|c|}{$\begin{array}{l}\text { DV: short-term } \\
\text { circumvention }\end{array}$} & \multicolumn{2}{|c|}{$\begin{array}{l}\text { DV: long-term } \\
\text { circumvention }\end{array}$} & \multirow[t]{2}{*}{ Test of coeff. diffs. } \\
\hline & $\beta$ & S.E. & $\beta$ & S.E. & \\
\hline \multicolumn{6}{|l|}{ Controls } \\
\hline Constant & $2.44^{* *}$ & 0.17 & $2.28^{* * *}$ & 0.19 & n.s. \\
\hline Age & $0.02^{*}$ & 0.01 & 0.00 & 0.04 & n.s. \\
\hline Gender $($ male $=0$, female $=1)$ & -0.02 & 0.08 & $-0.07^{*}$ & 0.03 & n.s. \\
\hline \multicolumn{6}{|l|}{ Primary independents } \\
\hline Perceived misfit (Likert-type 1-7) & $0.06^{* *}$ & 0.01 & $0.26^{* *}$ & 0.02 & $p<0.01$ \\
\hline Perceived ease-of-circumvention (Likert-type 1-7) & $0.09^{* *}$ & 0.02 & $0.08^{* *}$ & 0.01 & n.s. \\
\hline \multicolumn{6}{|l|}{ Interaction term } \\
\hline Perceived misfit $\times$ ease-of-circumvention & $0.022^{* *}$ & 0.006 & 0.00 & 0.63 & $p<0.01$ \\
\hline$\Delta R^{2}$ (on addition of interaction term) & $0.051^{* *}$ & & 0.012 & & \\
\hline Final $R^{2}$ & $0.502^{* *}$ & & $0.675^{* *}$ & & \\
\hline
\end{tabular}

Note. Since no other preliminary controls are significant in the regressions, either w.r.t. total model $R^{2}$ or factor coefficients, their numbers are excluded from this table. ${ }^{\star \star} p<0.01$, one-tailed, ${ }^{*} p<0.05$, one-tailed. 


\section{Table 5 GLS regressions for relevance of past supervisory experience}

\begin{tabular}{|c|c|c|c|c|}
\hline & \multicolumn{4}{|c|}{ DV: long-term circumvention } \\
\hline & \multicolumn{2}{|c|}{ Binary treatment effects } & \multicolumn{2}{|c|}{ Perceived context effects } \\
\hline & $\beta$ & S.E. & $\beta$ & S.E. \\
\hline \multicolumn{5}{|l|}{ Controls } \\
\hline Constant & $3.35^{* *}$ & 0.21 & $3.18^{* *}$ & 0.33 \\
\hline Age & 0.00 & 0.02 & -0.02 & 0.05 \\
\hline Gender $($ male $=0$, female $=1)$ & $-0.05^{*}$ & 0.02 & $-0.09^{*}$ & 0.04 \\
\hline \multicolumn{5}{|l|}{ Primary independents } \\
\hline Misfit $($ low $=0$, high $=1)$ & $1.75^{* *}$ & 0.32 & & \\
\hline Perceived misfit (Likert-type 1-7) & & & $0.24^{* *}$ & 0.08 \\
\hline Ease-of-circumvention $($ low $=0$, high $=1)$ & $0.41^{* *}$ & 0.15 & & \\
\hline Perceived ease-of-circumvention (Likert-type 1-7) & & & $0.06^{* *}$ & 0.03 \\
\hline \multicolumn{5}{|l|}{ Interaction term } \\
\hline Misfit $\times$ ease-of-circumvention binaries & 0.03 & 0.57 & & \\
\hline Perceived misfit $\times$ ease-of-circumvention & & & 0.00 & 0.23 \\
\hline \multicolumn{5}{|l|}{ Post-hoc experiential control } \\
\hline Past supervisory experience (staff count) & $-0.04^{*}$ & 0.02 & $-0.05^{*}$ & 0.02 \\
\hline$\Delta R^{2}$ (on addition of past supervisory term) & $0.039^{*}$ & & $0.031^{*}$ & \\
\hline Final $R^{2}$ & $0.683^{* *}$ & & $0.706^{* *}$ & \\
\hline
\end{tabular}

Note. Since no other preliminary controls are significant in the regressions, either w.r.t. total model $R^{2}$ or factor coefficients, their numbers are excluded from this table. ${ }^{* *} p<0.01$, one-tailed, ${ }^{*} p<0.05$, one-tailed.

\section{Fig. 1. Relationship between discomfort with prescribed rules and variation in processing.}

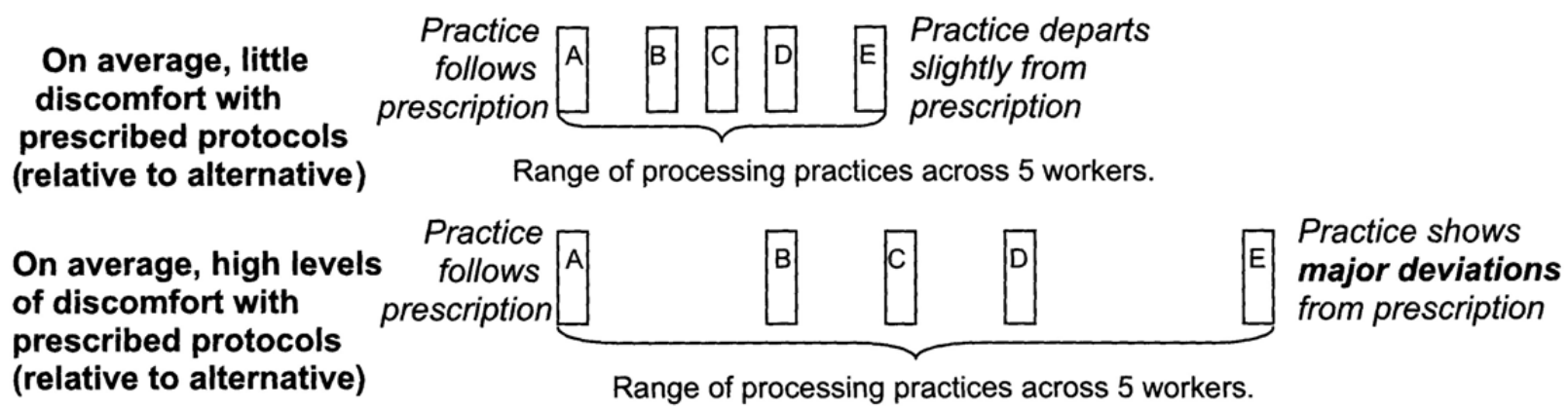


Fig. 2. Implication of strong-rule structures on the variation in processing practices.

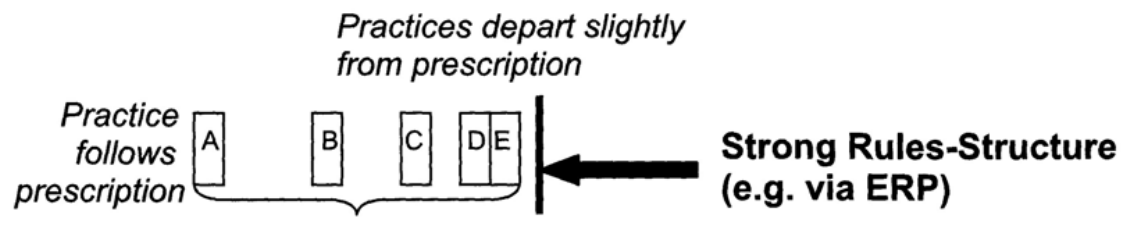

Range of processing practices across 5 workers when strong rule-structures force compliance with prescribed protocols; As long as such rule-structures continue to suppress alternatives the impact on practice may be largelyindependent of the average level of discomfort with prescription.

Fig. 3. Contingent role of perceived misfit, ease of circumvention and time on variation in practice.

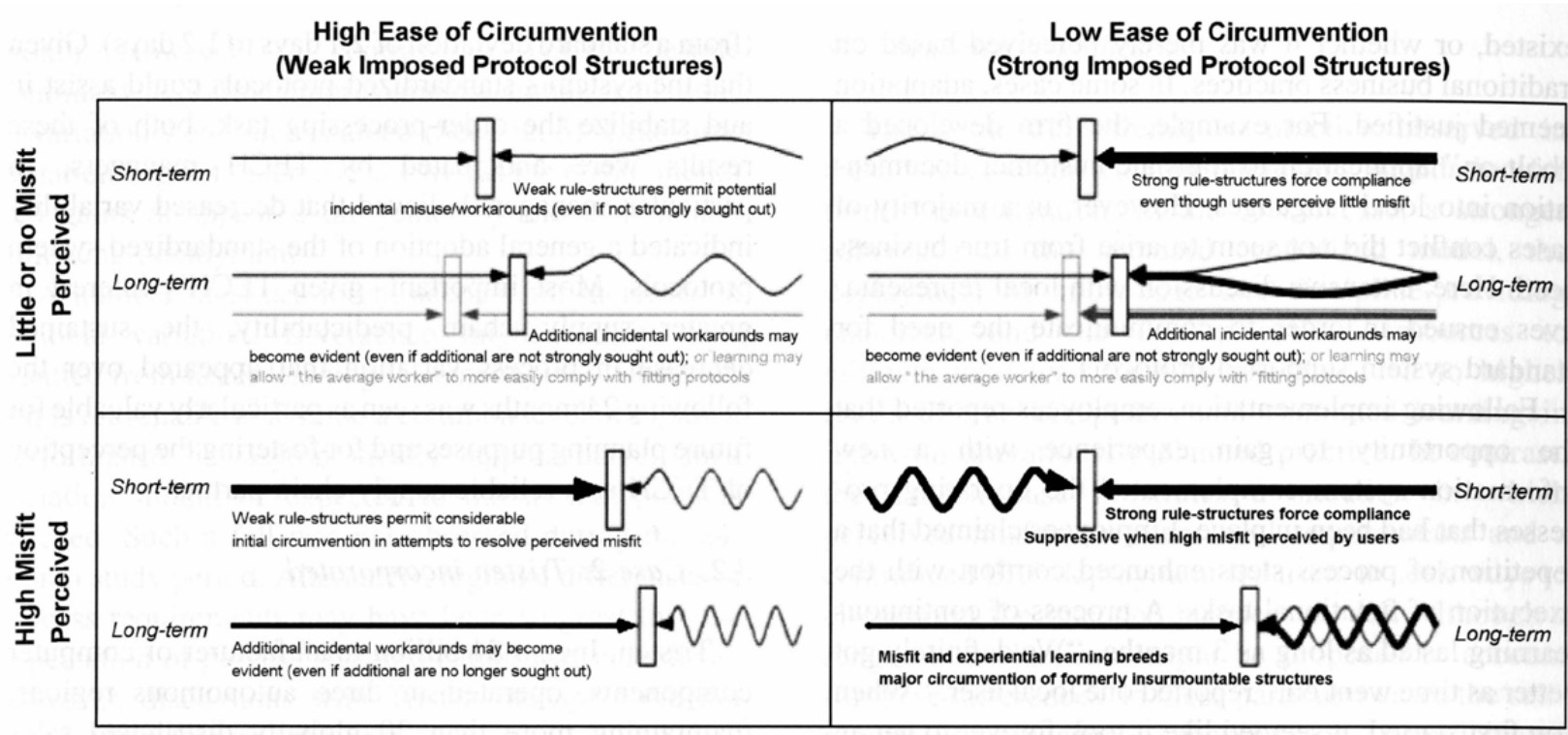


Fig. 4. Operational convergence trends after implementation at $\mathrm{TECH}$.

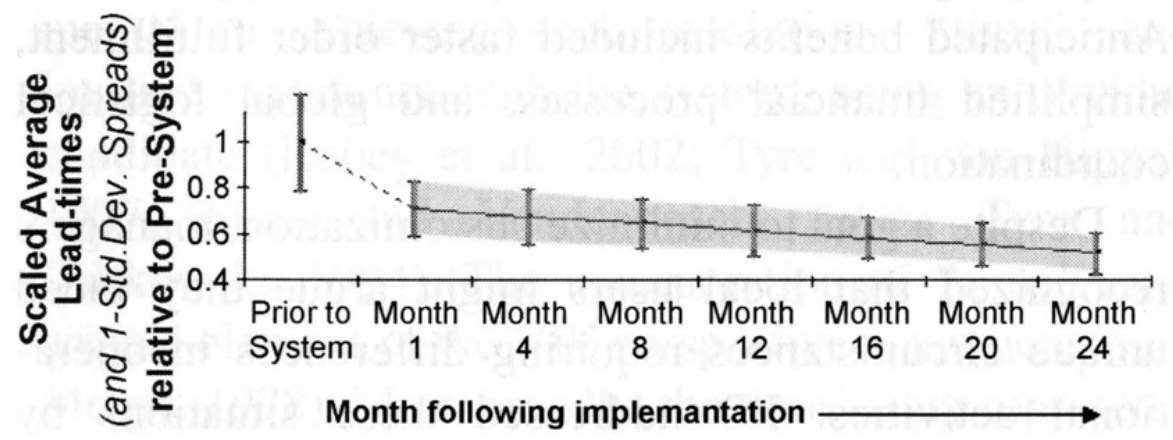

Fig. 5. Operational divergence trends after implementation at Tristen.

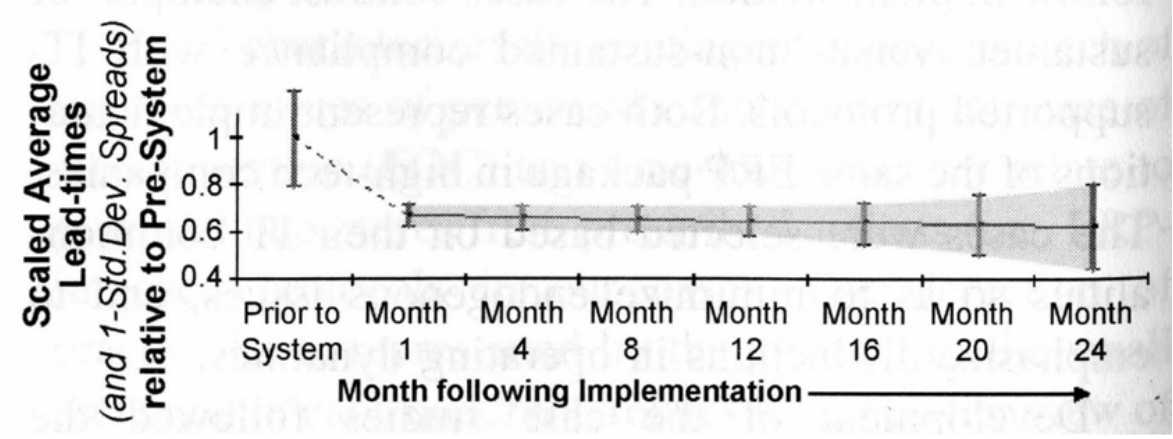


Fig. 6. Simple effect comparisons for each combined treatment and respondent reaction.
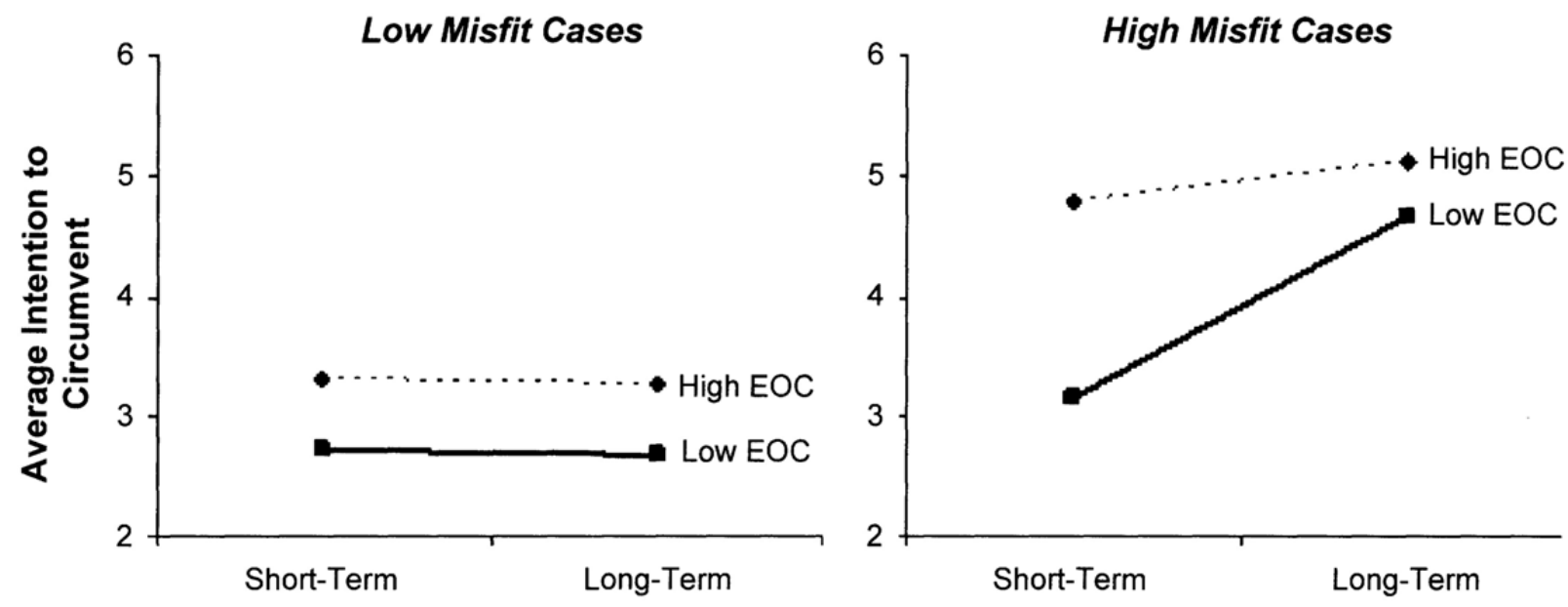

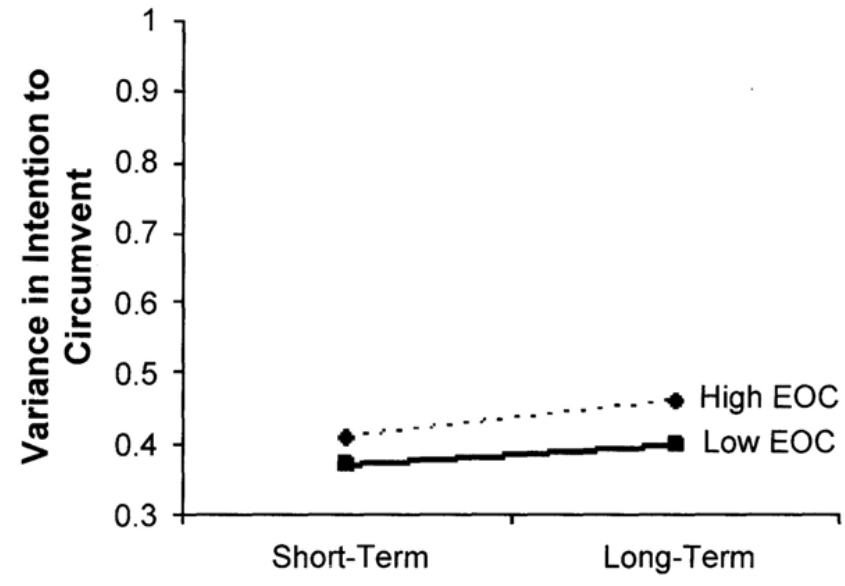

Time Frame for Intentions

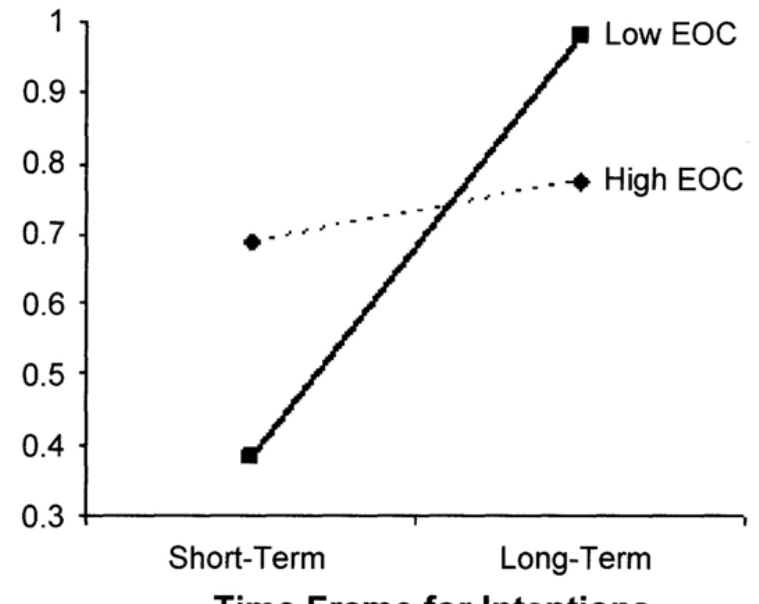

Time Frame for Intentions 
Fig. 7. Simple effect comparisons for past supervisory experience on long-term intentions given treatments.
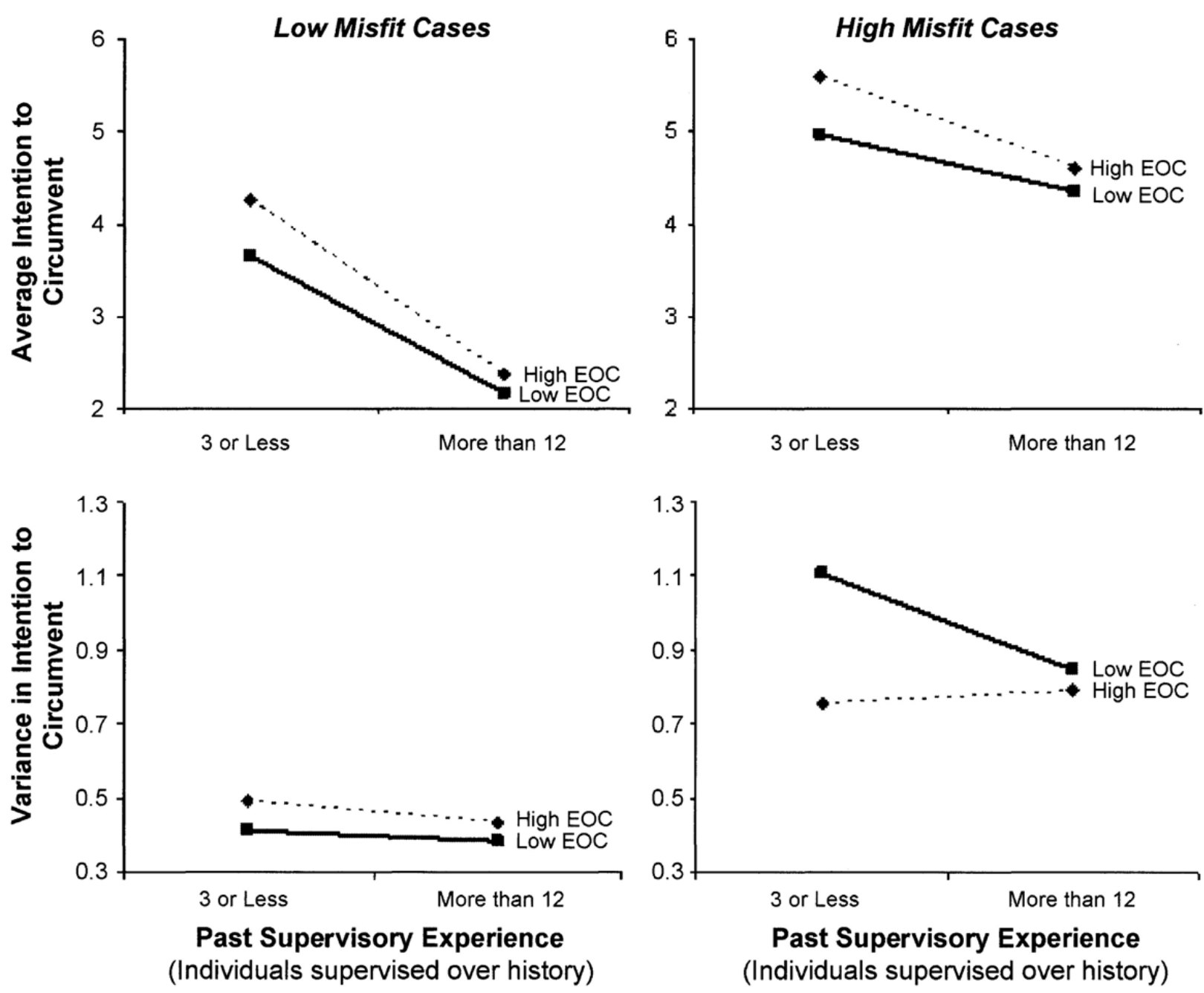